\title{
The Current Procedural Terminology and the Medicare Resource Based Relative Value Scale in neurosurgical practice
}

\section{Richard A. Roski, M.D.}

Quad City Neurosurgical Association, Davenport, Iowa

Changes in healthcare have made it increasingly more important for neurosurgeons to understand the economic pressures that effect their reimbursement. Two fundamental concepts that are of the greatest importance are the Current Procedural Terminology coding and the Medicare Resource Based Relative Value Scale. The impact of these two entities on neurosurgical reimbursement in analyzed in this review.

Key Words * Current Procedural Terminology coding * Resource Based Relative Value Scale * reimbursement * managed care

The federal government and many managed care organizations (MCOs) have done a great deal over the past several decades to significantly influence the economics of today's neurosurgical practice. It is now more important than ever that neurosurgeons understand the significance of proper Current Procedural Terminology (CPT) coding and the continually evolving changes in the Medicare Resource Based Relative Value Scale (RBRVS) if they wish to comprehend fully the economic changes taking place within their practices. These issues are important because neurosurgeons need to understand changing reimbursement and because there are growing concerns over charges of reimbursement fraud and abuse. A better understanding of the CPT process and the Medicare RBRVS will help neurosurgeons maneuver through the difficult economic times ahead.

In 1966 Physicians CPT coding was developed and first published by the American Medical Association in response to the increasing need for standardized terminology and clarity so that physicians and other healthcare providers could describe their work for purposes of billing health insurers. This was spearheaded by Medicare, which had been enacted into law in 1965. With rare exception, the federal government, state health agencies, insurance companies, and MCO plans now require physicians to describe their work using the American Medical Association's CPT coding system. The system not only assigns- a five-digit numerical code to surgical work but to patient evaluation and management (E/M) services as well. Proper use of CPT coding can help maximize the reimbursement to a physician; however, misuse can make physicians vulnerable to charges of fraud and abuse. Understanding the specifics of CPT coding has never before had such important consequences to practicing neurosurgeons.

The process of CPT coding for operative procedures is by no means an exact process. Some descriptors 
of CPT codes are somewhat nebulous and open to various interpretations. New operative procedures are not included in the CPT book until they have been well established across the country and often times do not appear until several years after the operative procedure has been in general use. Based on the short descriptor that appears in the CPT book, it is often difficult to know what specific operative components are included in each specific CPT code. These ambiguities create opportunities for misuse and abuse within the CPT coding system. In the past, understanding the CPT coding system helped physicians maximize reimbursement. If a physician improperly coded an operation on the insurance billing form, the claim might be incorrectly paid or simply denied. Inaccurate coding did not leave the neurosurgeon facing any severe repercussions. This, however, has changed. With more frequent computer screening of operative claims, the federal government as well as many insurance companies now question whether miscoded claims are deliberate attempts to defraud the government or the insurance carrier. Physicians face not only financial penalties for improper coding but civil charges as well. Insurance companies that mistakenly paid for improperly coded claims, when finally aware of the inaccuracies, now demand repayment of monies incorrectly paid to physicians. Concern over fraud and abuse charges is most dramatically illustrated in issues related to E/M coding. The Health Care Financing Administration (HCFA) has been very concerned about its ability to review physicians' charts adequately to ensure that the E/M services for which they are providing reimbursement, have been properly documented. A very large part of the Medicare budget is paid out for office visits, consultations, hospital admissions, and other E/M services. Most subgroups of the E/M codes have levels of service that range from 1 to 5, with higher payment made to the higher levels of service. The HCFA has been interested in developing specific guidelines to help physicians correlate the documentation of the work with the various levels of $\mathrm{E} / \mathrm{M}$ codes to facilitate its process of office reviews. The most recent $\mathrm{E} / \mathrm{M}$ documentation guidelines published by HCFA were finally rejected; however, the process continues and some slightly altered formal guidelines will come out within the next year. Although the recent documentation guidelines were rejected, the basic requirements for the various levels of E/M codes remain in effect. Physicians are still held accountable for proper documentation in their charts to substantiate the particular levels of service for which they have billed. For physicians in general, this is the largest area of potential exposure for review by both Medicare and MCOs. Therefore, physicians must understand the documentation requirements that are still in effect for E/M codes, and should periodically review their own office charts to ensure that the documentation present in the patient record coincides with the level of service that has been billed.

Although the CPT process has helped to define services rendered within the healthcare system, it is, by itself, a recording instrument and does not directly determine physician reimbursement. Fee schedules for physician services have historically been developed by the individual practitioner. Over time, fee schedules have been developed by insurance companies and MCOs to simplify contracting with physicians. Since 1992 the Medicare fee schedule has come to dominate the economic scene in healthcare. In 1987, the HCFA contracted with Professor William Hsaio at the Harvard School of Public Health to develop the RBRVS, which the HCFA then used as the basis for the Medicare fee schedule first published in 1992. Several changes have occurred since the initial Medicare fee schedule was published: 1) the RBRVS relative-value scale has been implemented by many insurance carriers and MCOs around the country; 2) many insurance companies have slowly started to push reimbursement rates toward and even below Medicare maximum-fee guidelines; and 3) with an increasing effort by the HCFA to improve reimbursement for primary care physicians, the reimbursement for E/M services has continued to increase at the expense of the fees for surgical procedures. These changes exert an important influence on the economics of neurosurgical practice. Just as Medicare attempted with Medicare fee 
schedule to control physician costs, many insurance companies and MCOs have also had difficulty trying to control payments for physician services. Although the CPT coding system has allowed them to better define the physician services, the fees being charged have historically varied dramatically around the country and even within a small region. Even establishing "usual and customary charges" or "average regional charges" only brought the surgical fees to within a range of consistency; they did not provide firm, fixed numbers that would allow insurance companies to make better long-term cost projections. The RBRVS became an important tool that made it easier for insurance companies to move toward a single-fee schedule for all CPT-coded physician services. This helped to eliminate gradually their need for individually contracting a set of surgical fees with every contracting physician. With the publication of the RBRVS, insurance companies believed they had a relative-value scale that had some degree of national acceptance and that they could use to develop their own fee schedule.

With the original work done by Professor Hsaio in developing the RBRVS, the relativity within the physician work component of the RBRVS scale was fairly reasonable. The immediate concern for physicians was what the dollar-conversion factor would be used to apply the fee schedule to a particular MCO plan or insurance company. Over time insurance companies have come to realize that if physicians accept Medicare payment for treating the elderly, then they would accept that payment for other patients as well. Many insurance companies have begun to adopt the Medicare fee schedule as their fee schedule. In several places around the country, managed care contracts are based on fees below the present Medicare levels. Although the intended Medicare fee schedule for reimbursement was understandable at its onset, its adoption by most of the healthcare industry has now produced significant economic difficulty for physicians. By providing an easy tool for insurance companies to develop fee schedules, the HCFA has provided them the means to ratchet down physician fees slowly. I am sure that all physicians have felt the pressure from decreasing reimbursement because of use of the RBRVS fee schedule.

Other changes have taken place within the RBRVS fee schedule, with may have even greater importance to practicing neurosurgeons. When the Clinton administration took office, there was much discussion about healthcare reform and the need for improving reimbursement to primary care physicians. Because primary care physicians almost exclusively use E/M codes for their services, the issue became one of how to improve reimbursement for E/M codes relative to surgical procedures. The physician work values in the RBRVS were sufficiently well established and accepted that changing them as a means of increasing payment to primary care physicians would have been difficult. Moreover, in the context of budget neutrality as imposed by the HCFA wherein all annual changes in physician reimbursement are limited to no more than $\$ 20$ million, the only way to increase payments to primary care physicians would be to reduce payments to other groups such as surgeons. This combination of factors led primary care physicians to lobby the administration and Congress to engineer a plan to redistribute payments for practice expenses from surgeons to primary care physicians. The practice-expense relative value units (RVUs) comprise approximately $40 \%$ of the total value of any surgical CPT procedure code, whereas malpractice accounts for only approximately $7 \%$ of the total. As it turns out, changing the practice-expense values has created the means by which the HCFA plans to change the value for CPT codes; this will have a direct effect on increasing the reimbursement for E/M services. Although the HCFAs attempts to change drastically the practice-expense component of the RBRVS were thwarted in 1997, significant changes proposed for 1999 have been published in the Federal Register. Table 1 shows the changes in RVUs from 1998 and those proposed for 1999 along with the percentage of change. 


\begin{tabular}{|c|c|c|c|}
\hline \multicolumn{4}{|c|}{\begin{tabular}{|c|} 
TABLE 1 \\
CURRENT 1998 AND PROPOSED 1999 CHANGES IN RVUS
\end{tabular}} \\
\hline \multirow[b]{2}{*}{ CPT Code } & \multicolumn{2}{|c|}{ RVU nonfacility } & \multirow{2}{*}{$\begin{array}{l}\text { Percentage } \\
\text { of } C \text { tange }\end{array}$} \\
\hline & 1998 & 1999 & \\
\hline 99213 & 1.13 & 1.25 & 11 \\
\hline 99214 & 1.71 & 1.98 & 16 \\
\hline 99243 & 2.79 & 3.23 & 16 \\
\hline 99244 & 3.92 & 4.49 & 15 \\
\hline 99245 & 5.28 & 5.71 & 8 \\
\hline 61510 & 60.39 & 50.75 & -16 \\
\hline 61700 & 87.88 & 85.56 & -3 \\
\hline 63030 & 28.01 & 23.54 & -16 \\
\hline 63047 & 35.16 & 29.55 & -16 \\
\hline 63075 & 40.19 & 35.4 & -12 \\
\hline
\end{tabular}

These proposed changes will have a significant negative impact on reimbursement for neurosurgical services and make it important for physicians to analyze more carefully how their practice time is utilized. Many years ago, office visits were considered a peripheral part of a surgeon's practice, because a majority of reimbursement came from performing operative procedures. Gradually the importance of reimbursement for office visits and consultations has increased. Pelofsky and Roski conducted a study 5 years ago for the Joint Officers of the American Association of Neurological Surgeons and the Congress of Neurological Surgeons in which they evaluated the percentage of RVUs of work within neurosurgical practice that were done for E/M services compared with surgical services. It was found that $26 \%$ of the RVUs were done performing E/M services and 74\% were done performing operative procedures. That percentage has increased over the past several years, and with the proposed changes for 1999, the reimbursement for E/M services will probably comprise 30 to $35 \%$ of the total reimbursement coming into a neurosurgical practice. Although the overall reimbursement is going to decrease with the proposed changes for 1999, physicians need to look carefully at time allocation within their practice to maximize the increased payments coming in for $\mathrm{E} / \mathrm{M}$ services. It becomes economically less feasible to leave operating days open when there is a slowing in the operative schedule, as opposed to using that time for consultation and other E/M services. Improved utilization of E/M services will help physician practices to somewhat offset the negative impact from the proposed RBRVS changes for 1999.

Physicians continue to be placed under increasing economic pressures from numerous sources, including Medicare and MCOs. The better physicians understand the procedures and processes being used by Medicare and the insurance industry, the better they will be able to plan for the economic variations in the future. It behooves all neurosurgeons to become better versed in the nuances of both CPT coding and the ongoing changes of RBRVS. Understanding these issues will help physicians prepare for the imminent economic changes in the coming years.

Manuscript received June 29, 1998.

Accepted in final form July 10, 1998.

Address reprint requests to: Richard A. Roski, M.D., Quad City Neurosurgical Association, P.C., 1351 West Central Park, Suite 2100, Davenport, Iowa 52804. 